\section{Aus dem Pharmakologischen Institut der Universität} in Halle a. S.

\section{Ueber Kollargol und Argentum colloidale. Von Erich Harnack.}

In einem kurzen Bericht über die fünfte Ausgabe des Deutschen Arzneibuches ${ }^{1}$ ) äußerte ich mich, abgesehen von anderen anerkennenden Worten, auch in folgender Weise: „Es muß hervorgehoben werden, daß es den Bearbeitern des Arzneibuches gelungen ist, in der sehr schwierigen Frage des gesetzlichen Wortschutzes für Arzneisubstanzen die rechtlichmateriellen Interessen mit den sachlich-ideellen zu vereinigen. Wenn der Arzt in der Rezeptur sich der geschuitzten populären Bezeichnungen bedient, so involviert das im allgemeinen etwas höhere Preise, aber die wissenschaftlichen Namen sind teilweise durch ihre Länge unbrauchbar, und die Populärnamen gew ̈̈h ren eine volle Garantie, die betreffenden Substanzen stets in gleich bleibender Beschaffenheit zu erhalten, obschon natürlich der Apotheker in allen Fällen verpflichtet ist, sich von der vorgeschriebenen tadellosen Qualität der von ihm abgegebenen Präparate zu überzeugen."

Wenn aber das Arzneibuch zur Prïfung solcher Qualität nichts Genügendes vorschreibt, wie dann? Können in solchen Fällen, wenn der Arzt aus Ersparnisgründen gezwungen wird, nach wissenschaftlichen Namen zu verordnen, nicht doch üble Folgen eintreten und sowolhl die materiellen Interessen von Produzenten als auch die ideellen des Arztes verletzt werden? r. Es muß eingeräumt werden, daß es dem Arzneibuch gegenüber jenen sehr schwierigen Verhältnissen und beim redlichsten Willen nicht überall gelungen ist, der Schwierigkeiten Herr zu werden und alle Interessenten, die überhaupt auf Rücksicht Anspruch haben, zu befriedigen. Das gilt namentlich von dem interessanten Artikel Argentum colloidale, das die Pharmakopoe zugleich als Kollargol bezeichnet.

Ein völlig reines kolloides Silber ist ja das Präparat nie, cs enthält stets gewisse Beimischungen je nach der Herstellungsart, sonst könnte es auch nicht nach Angabe des Arzneibuches unter dem Geruch nach verbrannten Haaren verkohlen; denn das beweist die Anwesenheit organischer Gele (eiweißartiger Körper). Ueber die Eigenschaften des Präparates wird wenig angegeben: das Hydrosol, die wäßrige kolloidale Lösung ( $2 \%$ ), ist undurchsichtig, im auffallenden Licht trübe, wird bei reichlichem Verdünnen durchsichtig klar, bleibt aber im auffallenden Licht trübe. Außerdem wird noch das Verhalten gegen Mineralsäuren, gegen verdünnte und gesättigte Kochsalzlösung, sowie das Verhalten beim Glühen erwähnt. Das ist alles.

Die Produzentin des „,Kollargols“, die bekannte chemische Fabrik v. Heyden, lenkte nun meine Aufmerksamkeit in einem dankenswerten Schreiben auf folgende Unstände:

,Der Artikel Argentum colloidale enthält keine Löslichkeitsvorschriften und schreibt auch keinen Mindestgehalt an Silber vor. Wie bekannt sein dürfte, bezieht sich die ganze vorhandene Literatur ïber Argentum colloidale auf das ,Kollargol", ein Präparat, das mindesten $75 \%$ (genauer $78 \%$ ) Silber enthält und bis 7. $5 \%$.in Wasser ziemlich leicht löslich ist. Gerade diese beiden Punkte: Lösliclıkeit und Silbergehalt, sind es, die bei der Darstellung die größten Schwierigkeiten bereiten. Sie sind aber auch von großer Bedeutung für die praktische Anwendung. Es ist ohne weiteres klar, daß eine z. B. 1 \% ige Kollargollösung in der Wirkung verschieden ist von der $1 \%$ igen Lösung eines Argentum colloidale von niedrigerem, unter Umständen sogar viel niedrigereın Silbergehalt. Ebenso klar ist es, daß die intravenöse Injektion der $5 \%$ igen Lösung eines nur $\% .11 \%$ olıne größeres Sediment

1) Diese Wochenschrift 1911, No. 5. löslichen Argentum colloidale niclit so gefahrlos ist wie diē vielfach geiibte intravenöse Einspritzung einer 5\% igen Kollargollösung. Da das Arzneibuch keine Vorschriften in dieser Hinsicht gegeben hat, so kann also ein Argentum colloidale sehr wohl den Anforderungen des Arzneibuches vollständig entsprechen und trotzdem therapeutisch minderwertig, unter Umständen sogar gefährlich sein. Besondere Erwähnung verdient noch in diesem Zusainmenhange die Anwendung dieser Präparate zu röntgenographischen Zwecken, da ja die Intensität des Schattens ausschließlich von dem Silbergehalt der betreffenden Lösung abhängt.

Nun schließt aber jeder Leser des Arzneibuclss aus der Fassung der Ueberschrift, daß die Präparate Argentuın colloidale und Kollargol identisch seien. Bestimmte Krankenkassen haben in dieser Voraussetzung ihren Aerzten bereits vorgeschrieben, statt Kollargol nur noch Argentum colloidale zu verordnen. Nirgends steht $z u$ lesen, obgleich doch zweifellos dafür Platz. gewesen wäre, daß Kollargol nicht ein jedermann zur Verfügung stehender Name für kolloidales Silber, sondern die geschütıte Bezeichnung für ein ganz spezielles Präparat ist. Ganz abgesehen davon, daß durch das Verfahren des Arzneibuches aus geschützten Warenzeichen chemische Bezeichnungen gemacht werden, was nicht im Interesse der Warenzeicheninhaber liegt, so werden zweifellos auch viele Apotheker und sonstige Konsuinenten bona fide zu Warenzeichenverletzungen veranlaßt.

Während früher die Nachahmer des Kollargol gezwungen waren, ein dem Kollargol möglichst nahe kommendes Produkt zu liefern, was ihnen allerdings bis jetzt noch nicht gelungen ist, wird ihnen jetzt durch die außerordentlich laxen Anforderungen des Arzneibuches die Sache erleichtert. Die Nachahmer brauchen jetzt nur noch ein den Anforderungen des Arzneibuches entsprechendes Produkt zu liefern, und lat das Arzneibuclı für dessen Einfiïrung auch schon gesorgt.

Die Aufnahme des Artikels "Argentuin colloidale etc." liat also folgende Konsequenzen: 1. Für den Arzt: Er riskiert, wenn er Argentum colloidale verschreibt, ein minderwertiges, unter Umständen lebensgefährliches Produkt zu erhalten, mit dem er nicht in der Lage ist, die vom Kollargol her gewohnten Wirkungen zu erzielen. 2. Für den Patienten: . . . 3. Für den Fabrikanten des Kollargols: Von der Verordnung in Krankenkassen und in manchen Krankenhäusern, wie auch vielfach in der Rezeptur ist Kollargol ausgeschlossen und ersetzt durch Präparate, die wegen geringen Silbergehaltes und wenig sorgfältiger Darstellung zu billigen Preisen angeboten werden können.

Diesem Manko gegenüber steht nicht der geringste Nut\%en für die Allgemeinheit oder für den Apotheker. Den einzigen Nutzen haben die Fabrikanten von Nachahmungen, für die das Arzneibuch eine kostenlose Propaganda gemacht hat."

Ich verdanke der Firma von Heyden auch die Mitteilung über die Zusammensetzung der folgenden sechs im Handel als „Argentum colloidale" bezeichneten Präparate.

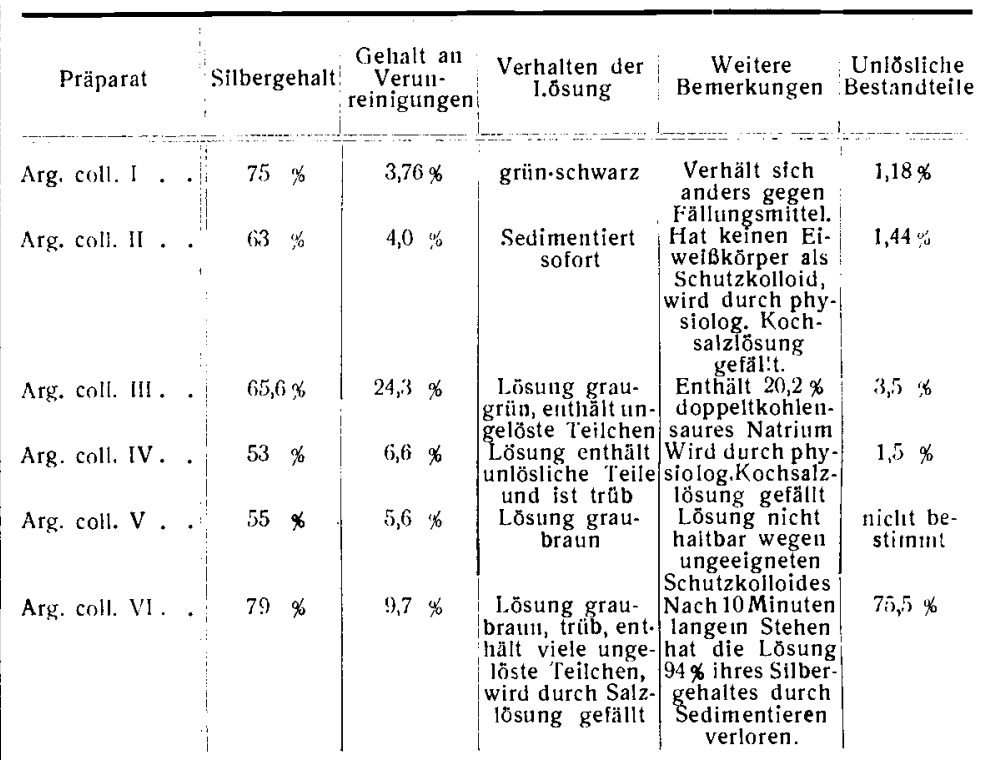

Das „Kollargol“ dagegen besteht aus mindestens $75 \%$ kolloidem Silber und etwa $25 \%$ Eiwei $\beta$ als sogenanntem Schutzkolloid mit einer der elektrischen Ladung der Kollargolteilchen gleichnamigen Ladung. Die letztere ist ja merkwürdigcrweise bei den kolloiden Metallen im Gegensatz zu den gewöhnlichen Metallionen negativ. Das gleichnamig geladene Kolloid erhöht die Widerstandsfähigkeit des Hydrosols gegen fällende Einflüsse durch Elektrolyte, das entgegengesetzt geladene tierische Eiwei $\$$ fällt dagegen selbst. Olne Schutzkolloid wird das Hydrosol des Goldes ja bekanntlich durch den Zusatz der geringsten 
Spur cines Elektrolytcs (Kochsal\%, Säurcn ctc.) sofort verändert \%mü̈chst durch Farbcıwcchsel, dann abcr allmählich dic Scdiuncuticrung in sichtbarer Gestalt eingeleitet. Dic nur mit Hilfe des Ultramikroskops sichtbaren winzigen Teilchen haben iu Hychosol cinc unguncin rapide Bewcgnng, die um so träger wird, je nechr dic S'edintenticrung sich einstellt. Das schr merkwiirdige Verhalten lıöehst verdiiınter Kollargollösung gegen clektrisch verschieden geladcne: organische Kolloide: ist zm! Reaktion anf deren clektrische Ladnng nencrdings benutzt

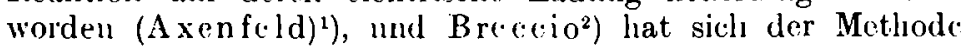
bedicut, "un \%u entscheiden, ob cin scröscr Lrguß transsudativen odcr cutzïndlichen Urspiungs sei. Fisteres wirkt auf dic Kollargollösung (1 : 15000) fällend, let\%teres fällungverhiudernd.

Dic: obige Tabelle reigt allerelings in angenfälliger Weise, wic schr sich jenc Handelspräparate des Argentunı colloidalc gcrade in Betreff der wichtigsten und ansschlaggebenden Eigenschaften rom richtigen ,Kollargol" unterscheiden, da nicht nur der silbergehalt bedentend variiert, sondern auch das Verhalt '"l der lössungen weschtlich verschieden ist. Selbst die Färbung der Hydrosole ist verschieden, was sich num durch Vermureinignıgen erklärt. Die Kollargollösung ist im auffallendcn licht tiefbraun mind triibe, im durchfallenden rotbram und klar, aber nicnıals gran oder grün. Am schlinınsten er'seheinc!n dic Präparatc 111 und VI, wälırend 1 am besten ist.

Wenn es anch dic Stimme eines Interessenten ist. die hier Klage: (rhelbt, so mul man ihr doch rollkonmen beipflichten. Es liegt eine luieke in Arrneibuehe vor, und die Fassung des ne.u anfgenonınıenen Artikels, so wic sie vorliegt, hat in der 'lat dadurch, daß sie unwillkiirlich den Eindruck der Idcntität von Kollargol und Argentun colloidale hervorruft, den schion bestandenen Sehaden nicht merheblich vergrößert.

1)iese Lüuke wird allch von dem Kominentar \%ur Pharmakopoe ${ }^{3}$ ), über (len ieh kür\%lich in dieser Zeitselhrift referiert labe, einigetmalsen \%ugestanden. Fis heißt (lort: „1)ie vom Ar\%ncibnch angefihiten Identitiitsreaktionen gewährle-isten nur, daß Silber in kolloilaler form mit "inel Kiweißsubstan\% als Sehut\%kolloil vorlicgt." Aber stelbst letatere. trifft niclit ganz zu; denu die angegebene Reaktion (Verkohlen unter typischem (Geruch) bew(ist nur, duß Wiweiß zugegen, nicht aber, daß (s) das richtig gewithlte Schut/kolloid ist.

Man darf nicht vergessen, wie schr dic Schwierigkeit schon dadurch gesteigert wird, daß es sich hier nieht um cine durchweg cinheitliche Substanz. handelt und dak das Mittel in der Praxis aluf jede: Weise, stllbst intravenös, \%ur Anwendung gebracht wird. Hätte ınan es nit einem chemisch reinen Körpe\%u tun, so wäre ess ja nicht schwierig, gecignete Identitätsraktionen etc. vorzuschreiben. Hier liegt die Sache aber vicel komplizie lter: durch die notwendige: Anwescenheit von liweil wird anch das beste. Präparat nicht völlig frei vou ,.Verunreinigungen" sein, schon wegen der Aschebrstandteile des biweißes. In älteren Präparaten habe ich anch neist cin ge:wissc's Quantum von Anmonsalzen ge:funden.

Daß3 clas Arrneibueh neben dem wissensclaaftlichen Namen anch die populäre, gesetzlich gesehützte: Be\%eichnung anfiihrt, kann man ihm an sich nieht zum Vorwurf machon. Lis ist in zahlreichen an zlogen Fällen fa anch geschehen und konnte garnicht vermieden werden. Aber in diesenı gan\% eigenartig beschaffenen Falle sollte der Text des Artikels cinfach die Ligenschaften des praktisch bewährten Kollargols fordern und darnach also die einze. Inen Methoden der Prüfung in be'zung auf Silbergchalt, Farbe: und Figenschaften der Lösung, Verhalte:n gegen vereliinnte. und kon\%entrierte Elektrolyte cte. vorschreiben. Es hicße das durchans nicht ein Monopol statuicren, wohl aber die Produktion zwingen, keine dem, ,Kollargol" gegenüber ininderwertigen Präparate anf den Markt 7.u bringen.

Es liegt nicht in meiner Absicht, dic Indikationen fïr die: Anwendung des kolloiden Silbers anfzuzählen und kritisch zu wïrdigen; tatsächlich ist es bercits sehr vielfach, in recht verschicdencn Formen (Tablette, lösung, Salbe, Suppositorien, Stäbchen) und anf allen möglichen Applikationsstellen angewendet worden. Es hat sich als Antizymotikmm be:währt und ist ein Katalysator wic: andere Mctalle. Bei der SchuB-

1) Zentralblatt für Physiologie 1909, No. 23.

*) Berliner klinisehe Wochensehrift 1910. No. 34.

3) 5. Ausgabe von Ansolmino und Gilg 1911, Bd 1, S. 260. wundenbehandhung im Kriege: nach den v. Borgmannschen Prinzijien hat e's vortreffliche Dienste gele istet. v. Ucttingen nahm in den russisch-japanischen Kricg nicht weniger als 20000 Kollargoltabletten (a 0,05$)$ mit, zog jedesmal mu dic. Schußöffnung einen klcbenden Mastixring, $u n$ chas Einstre $i c \cdot h e n$ bakterieller Kontaktkeime: \%u verhiiten, legte cinc Tablette: in die offenc Wunde und deckte mit keichtem sterilen Verbande. Die. Fifolge nuïssen unter den gegebenen Lmständen als merkwiirdig giinstig bercichnct werken. Anch in der Behandhung der Gonorrhoe hat sich das Kollargol nebern anderen moderncen Silbierpräparaten oinen guten Ruf clworben, und es ist dadurch bewiesen worden, daß pathogene. Mikroorganismen anch iu der Beriihrung mit solchen Hydrosolen, die doch gar keine: wirklichen Lösungen sind, schr wenig giinstige Be-dingung(-n) fïr ihre Weitcrentwicklung finden.

Den kolloiden Mctallen ist es ganz cigcmartig ergangen: als sie zucerst anftauchten, begegneten sic cincer starken Skepsis, und ich mul gestchen, dak ich anch nicht frei von diescer war, wcil eben dic Anwesenhe it frendartiger Sulstanzen in den

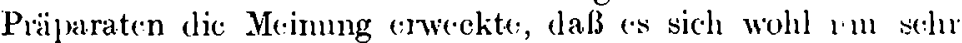
sillserteiche organische Verbindungen, nicht ale: mu Vietall im kolloiden Kastande handelte. Das Urteil anderte sieh indes bald. Die: Chemice und Physik der kolloiden Körper iilscrlanıt, zu denen e.ben die kolloiden Mctalle anch gehöre'n, "nt wickelte’n sich rapide zn ciner e.igenen Disziplin, mel zwar e.ine. Disziplin, dic fïr biologische und vielfach anteh fiir pharmakologische Fragen eine große: 'lragweite besitzt. So ist alhuählich anch das therapentische: Interesse an den kolloiden Mitallen gewachsen: in Frankicich wird die Anwendung des kolloiden Quceksilbers gegen Lues auf Grund \%ahlreicher Wrfahrungen warn empfohlen und häte vielleicht anch bei uns nuchr Beachtung gefunden, wenn sich nicht dic organischen Arsenverbindungen plöt\%lich in den Vordergrund gestellt hätten. Noch hat unser Aroneibuch das ,Hydrargyrmm colloidale." nicht aufgenoume'n, sollte es aber ge:sehe-hen, so werelen hoffentlich genanc Vorschriften iibel l'rüfung und Feststellung der Eigenscliaften gegeben werden, wic; wir sic fiir das kolloide: Silber als wiinschenswert erkannt haben. 